\title{
RACAH RECOUPLING MATRICES FOR SYSTEM OF TWO FREE ELECTRONS CONFINED TO SINGLE STAR
}

\author{
T. LULEK \\ Institute of Pliysics, A. Mickiewicz University \\ Matejki 48/49, 60-769 Poznan, Poland \\ AND J. KLOS \\ Institute of Molecular Physics, Polish Academy of Sciences \\ Smoluchowskiego 17/19, 60-179 Poznan, Poland \\ (Received September 19, 1995; in final form July 17, 1996) \\ Racah-Wigner type of calculus is adapted to the system of two free \\ electrons in a cubic box, confined to a single star $p 00$ of quasimomentum. \\ Various coupling schemes ( $L S$-like, $j j$-like, and transitive) have been re- \\ viewed in analogy to the case of a free atom. Racah recoupling matrices are \\ calculated explicitly, and thcir quantum-mechanical meaning is exposed in \\ tcrms of octahedral multipoles.
}

PACS numbers: 02.20.-a, 31.10.+z, 75.10.Lp, 75.20.-g

\section{Introduction}

We observe recently an increasing interest in electronic properties of systems with a finite number of electrons, like nanometer-scale magnets [1], quantum dots [2], quantum Ilall effect [3], or high temperature superconductivity [4]. Finite volume of such systems, and small number of electrons, justify a Racah-Wigner type of approach which has proved to be successful in the case of free atoms or ions [5]. It becomes interesting to establish conservation laws, good quantum numbers, classification of states, determination of energy level structure, selection rules etc. for various standard model systems, like free, bound, or itinerant electrons in finite cavities with different geometries. It encourages us to attempt to an adaptation of standard techniques of Racah-Wigner calculus to such extended $N$-electronic systems [6]. In this spirit, we examine in the present paper the particular notion of Racal recoupling matrices [7] (or their symmetrized version, usually referred to as the $3 n j$-symbols), within the model of free electrons in a periodic cubic box. We restrict the considerations to a simple case of $N=2$ electrons, which are confined to a single star $(p 00)$ of quasimomentum. In due course we expose analogies with the theory of atomic spectra. 


\section{Electronic spaces}

a) The single-electron space has the form

$L=B \otimes W$,

where

$$
B=\operatorname{lc}_{\mathcal{C}} \varphi
$$

is the positional space, spanned on an orthonormal basis labelled by arms of the star of quasimomentum

$$
\varphi=\{| \pm p, 0,0\rangle,|0, \pm p, 0\rangle,|0,0, \pm p\rangle\},
$$

where $\left|n_{x}, n_{y}, n_{z}\right\rangle, n_{x}, n_{y}, n_{z} \in Z$, denotes the orbital with the quasimomentum $k=(2 \pi / L)\left(n_{x}, n_{y}, n_{z}\right), Z$ is the ring of integers, $L$ is the length of an edge of the cube,

$$
W=\operatorname{lc}_{\mathcal{C}}\left\{m_{s}= \pm 1 / 2\right\}
$$

is the spin space of a single electron, and the symbol $l_{\mathcal{C}}$ denotes the linear closure, i.e. the space over the field $\mathcal{C}$ of complex numbers, spanned on an appropriate set of states. The atomic counterpart of (1) consists in factorization of single-electron shell spaces to orbital and spin factors. In our case,

$$
\operatorname{dim} L=\operatorname{dim} B \cdot \operatorname{dim} W=6 \cdot 2=12 .
$$

We observe that our orbital analogue - the positional space $B$ is 6-dimensional, which is intermediate between atomic $d$ and $f$ electrons (with the dimension $2 l+1=5$ and 7 , respectively).

b) The two-electron space is determined by the Pauli exclusion principle, which yields that it is the antisymmetric square $L^{\left\{1^{2}\right\}}$ of the space $L$. Taking into account the factorization (1), we obtain

$$
L^{\left\{1^{2}\right\}}=(B \otimes W)^{\left\{1^{2}\right\}} \approx B^{1^{\{2\}}} \otimes W^{\{2\}} \oplus B^{\{2\}} \otimes W^{\left\{1^{2}\right\}},
$$

where the first and the second term in the right hand side of $(6)$ corresponds to triplet $(S=1)$ and singlet $(S=0)$ pairing, respectively. The dimensional check reads

$$
\operatorname{dim} L^{\left\{1^{2}\right\}}=15 \cdot 3+21 \cdot 1=66=\left(\begin{array}{c}
12 \\
2
\end{array}\right),
$$

i.e. the triplet and singlet manifold exhibits 15- and 21-fold orbital degeneracy, respectively. The atomic counterpart of (7) is

$$
10 \cdot 3+15 \cdot 1=45=\left(\begin{array}{c}
10 \\
2
\end{array}\right)
$$

and

$$
21 \cdot 3+28 \cdot 1=91=\left(\begin{array}{c}
14 \\
2
\end{array}\right)
$$

for $d^{2}$ and $f^{2}$ configurations, respectively. 


\section{Coupling schemes}

The factorization (1) of the single-electron space $L$ into the positional and spin factor spaces yields the same variety of possibilities of multiple couplings in construction of many-electron spaces as in the case of Wigner-Racah calculus for atoms. They involve $3 j, 6 j$ and higher order symbols, coefficients of fractional parentage, etc. We demonstrate here only the simplest case of two electrons.

The positional space $B$ is the carrier of a representation $R$ of the symmetry group $G$ of the box, which we assume hereafter to be the octahedral group $O_{h}$. In the case of a single star $\varphi$, this is the transitive representation $R^{O_{h}: C_{4 v}}$, with $C_{4 v}$ being the stabilizer of a single arm of the star (3). The corresponding decomposition

$$
B=\sum_{\lambda \in \tilde{O}_{h}, t \in \tilde{m}(R, A)} \oplus B^{A t}
$$

of the positional space $B$ into irreducible subspaces $B^{A t}$ points out the role of irreducible representations (irreps) $\Lambda \in \tilde{O}_{h}$ as analogs of the single-electron orbital angular momentum quantum number $l$. Ilere $\tilde{O}_{h}$ denotes the set of equivalence classes of irreps of $O_{h}$, which in the Mulliken notation reads

$$
\tilde{O}_{h}=\left\{A_{1 \mathrm{~g}}, A_{1 \mathrm{u}}, A_{2 \mathrm{~g}}, A_{2 \mathrm{u}}, E_{\mathrm{g}}, E_{\mathrm{u}}, T_{1 \mathrm{~g}}, T_{1 \mathrm{u}}, T_{2 \mathrm{~g}}, T_{2 \mathrm{u}}\right\},
$$

and $\tilde{m}(R, \Lambda)=\{t=1, \ldots m(R, \Lambda)\}$ is the set of repetition labels for multiplicity $m(R, \Lambda)$ of $\Lambda$ in $R$. In our case

$$
R^{O_{l}: C_{4 v}} \approx A_{1 \mathrm{~g}} \oplus E_{\mathrm{g}} \oplus T_{1 \mathrm{u}},
$$

so that the repetition label $t$ is redundant. The scheme defined by the basis

$$
\left|\Lambda_{1} \Lambda_{2} \Lambda \lambda, S, \Gamma \gamma\right\rangle
$$

corresponds to atomic $L S$ coupling for two electrons. The irrep $\Lambda$, which emerges from the Clebsch-Gordan decomposition

$$
\Lambda_{1} \otimes \Lambda_{2}=\sum_{\Lambda \in \tilde{O}_{h}} \oplus m\left(\lambda_{1} \otimes \Lambda_{2}, \Lambda\right) \Lambda
$$

of single-electron orbitals $\Lambda_{1}$ and $\Lambda_{2}$, is the analogue of atomic orbital quantum number $L, S$ is the same as for atoms $(S=1$ and 0 for spin triplets and singlets, respectively), $\Gamma$ corresponds to the total angular momentum $J$ and arises from

$$
\Lambda_{1} \otimes \Delta=\sum_{\Gamma \in \tilde{O}_{h}} \oplus m\left(\Lambda_{1} \otimes \Delta, \Gamma\right) \Gamma
$$

where $\Delta$ is an irrep of $O_{h}$ corresponding to $S\left(\Delta=A_{1 \mathrm{~g}}\right.$ and $T_{1 \mathrm{~g}}$ for $S=0$ and 1 , respectively), and $\gamma \in \tilde{\Gamma}$ is the degenerate basis of $\Gamma$, i.e. the counterpart of the $z$-projection $M_{J}$ of the total angular momentum $J$. Equation (12) can be also interpreted in terms of multipole expansion of plane waves. Six plane waves, labelled by arms of the star $\varphi$ can be rearranged into cubic partial waves, labelled by irreps $\Lambda$ entering (12). $\Lambda=A_{1 \mathrm{~g}}, E_{\mathrm{g}}$, and $T_{1 \mathrm{u}}$ is the cubic counterpart of a monopole $(l=0)$, quadrupole $(l=2)$ and dipole $(l=1)$, with $l$ denoting the rank of the parent spherical multipole. Correspondingly, Eq. (14) describes a superposition 
of single-electron cubic partial waves $\Lambda_{1}$ and $\lambda_{2}$ into the resultant cubic multipole $\Lambda$. Another scheme, namely

$$
\left|\Lambda_{1} \Gamma_{1}, \Lambda_{2} \Gamma_{2}, \Gamma w \gamma\right\rangle
$$

corresponds to atomic $j$ - $j$ coupling. Ilere, the octahedral group $O_{h}$ should be replaced by its double group $O_{h}^{\prime}$. We have

$$
\tilde{O}_{h}^{\prime}=\tilde{O}_{h} \cup\left\{E_{\mathrm{g}}^{\prime}, E_{\mathrm{u}}^{\prime}, E_{\mathrm{g}}^{\prime \prime}, E_{\mathrm{u}}^{\prime \prime}, U_{\mathrm{g}}^{\prime}, U_{\mathrm{u}}^{\prime}\right\}
$$

where the last set in the right-hand side of (17) corresponds to spinor irreps. The label $w$ distinguishes repeated irreps in the Clebsh-Gordan series for $O_{h}^{\prime}$.

A distinctive feature of our approach consists in a fact that the symmetry group $O_{h}$ acts in the positional space $B$ essentially as a permutation representation on the set $\varphi$ of arms of a star. An explanation of this fact in the midst of Racah-Wigner calculus relies on the Mackey theorem for transitive representations [8]. It allows a new coupling, called the transitive scheme, which is not present in the atomic case.

The Mackey theorem consists in the decomposition of the direct product of two constituent transitive representations into resultant transitive representations. The general formula is

$$
R^{G: H} \otimes R^{G: D}=\sum_{\omega \in \Omega} R^{G: L_{\omega}},
$$

where $H, D$ and $L_{\omega}$ are stabilizer's of the first and second constituent, and resultant representation, respectively, $\Omega$ is the set of double cosets of the group $G$ with respect to its subgroups $D$ and $H$, defined by the decomposition

$$
G=\bigcup_{\omega \in \Omega} D_{g_{\omega}} H
$$

with representatives $g_{\omega}$, and the stabilizer $L_{\omega}$ is given by the intersection

$$
L_{\omega}=D \bigcap g_{\omega} H g_{\omega}^{-1} \text {. }
$$

Mackey theorem is a transitive analogue of coupling of two angular momenta. In our case the Mackey theorem reads

$$
R^{O_{h}: C_{4 v}} \otimes R^{O_{h}: C_{4 v}}=2 R^{O_{h}: C_{4 v}}+R^{O_{h}: C_{1 h}},
$$

which corresponds to the direct product of the action of $O_{h}$ on the star $\varphi$. Pairs of arms of the form $(k, k),\left(k, k^{\prime}\right)$ with $k^{\prime} \neq \pm k$, and $(k,-k), k, k^{\prime} \in \varphi$, correspond respectively to the orbit $\omega=0,1$, and 2 of the resultant transitive representation in the right-hand side of Eq. (21). The orbits $\omega=0$ and $\omega=2$ are characterized by the stabilizer $C_{4 v}$, whereas the orbit $\omega=1$ corresponds to $C_{1 h}$. The transitive scheme is of the form

$$
|\omega \Lambda a \lambda, S M\rangle \text {, }
$$

where $\Lambda \in \tilde{O}_{h}$ is an irrep entering the resultant transitive representation $R^{O_{h}: L_{\omega}}$, and $a \in \tilde{m}(\omega, \Lambda)$ is the corresponding repetition label.

The label $\omega$ of the resultant transitive representations in Eq. (21) has a clear kinematical meaning: orbits $\omega=0,1$, and 2 gather pairs of momenta which are mutually parallel, perpendicular, and antiparallel, respectively. Each orbit $\omega$ gives rise to some irreps $\Lambda$ corresponding to appropriate octahedral partial waves. 
In order to complete the classification scheme, we need to point out restrictions imposed on each coupling scheme by the Pauli exclusion principle. It requires that the two-electron basis states should be antisymmetric under the transposition of electrons, i.e. they should be characterised by the irrep $\left\{1^{2}\right\}$ of the symmetric group $\Sigma_{2}$ acting on electrons. This restriction can be incorporated within each of the coupling schemes (13), (16), and (22).

The statistics $y_{S}$ of the spin part is

$$
y_{S}= \begin{cases}\{2\} & \text { for } S=1 \\ \left\{1^{2}\right\} & \text { for } S=0 .\end{cases}
$$

To determine the statistics $y_{0}$ of the orbital part in schemes (13) and (16), we have to consider two cases: (i) $\Lambda_{1} \neq \Lambda_{2}$, and (ii) $\Lambda_{1}=\Lambda_{2}$. In the former case the definite orbital statistics is established by putting

$$
\left|y_{0} \Lambda_{1} \Lambda_{2} \alpha\right\rangle=2^{-1 / 2}\left(\left|\Lambda_{1} \Lambda_{2} \alpha\right\rangle \pm\left|\Lambda_{2} \Lambda_{1} \alpha\right\rangle\right)
$$

which yields $y_{0}=\{2\}$ and $\left\{1^{2}\right\}$ for the upper and lower sign in (24), respectively. In the latter case the statistics $y_{0}$ is given by the unique decomposition

$$
\Lambda_{1} \otimes \Lambda_{1}=\Lambda_{1}^{\{2\}} \oplus \Lambda^{\left\{1^{2}\right\}}
$$

of the square of the irrep $\Lambda_{1}$ into the symmetric and antisymmetric parts, e.g.

$$
T_{2 \mathrm{u}} \otimes T_{2 \mathrm{u}}=T_{2 \mathrm{u}}{ }^{\{2\}} \oplus T_{2 \mathrm{u}}^{\left\{1^{2}\right\}}
$$

with

$$
T_{2 \mathrm{u}}^{\{2\}}=A_{1 \mathrm{~g}} \oplus E_{\mathrm{g}} \oplus T_{2 \mathrm{u}}, T_{2 \mathrm{u}}^{\left\{1^{2}\right\}}=T_{1 \mathrm{~g}} .
$$

The total statistics $y$ is given by

$$
y=y_{0} y_{S}= \begin{cases}\{2\} & \text { for } y_{0}=y_{S^{\prime}} \\ \left\{1^{2}\right\} & \text { for } y_{0} \neq y_{S^{\prime}}\end{cases}
$$

The Pauli exclusion principle admits only those states for which

$$
y=\left\{1^{2}\right\} \text {. }
$$

Statistics of the orbital part in the transitive scheme is determined as follows (cf. [9] for details of the orbit structure of the groups $O_{h}$ and $\Sigma_{2}$ )

$$
\begin{aligned}
& \left(R^{O_{h}: C_{4 v}}\right)_{\omega=0}=\left(R^{O_{h}: C_{4 v}}\right)_{\omega=0}^{\{2\}} \approx A_{1 \mathrm{~g}} \oplus E_{\mathrm{g}} \oplus T_{1 \mathrm{u}}, \\
& \left(R^{O_{h}: C_{14}}\right)_{\omega=1}^{\{2\}} \approx A_{1 \mathrm{~g}} \oplus E_{\mathrm{g}} \oplus T_{1 \mathrm{u}} \oplus T_{2 \mathrm{u}} \oplus T_{2 \mathrm{~g}}, \\
& \left(R^{O_{h}: C_{1 \mathrm{u}}}\right)_{\omega=1}^{\left\{1^{2}\right\}} \approx A_{2 \mathrm{~g}} \oplus E_{\mathrm{g}} \oplus T_{1 \mathrm{~g}} \oplus T_{1 \mathrm{u}} \oplus T_{2 \mathrm{u}}, \\
& \left(R^{O_{h}: C_{4 v}}\right)_{\omega=2}^{\{2\}} \approx A_{1 \mathrm{~g}} \oplus E_{\mathrm{g}}, \\
& \left(R^{O_{h}: C_{4 v}}\right)_{\omega=2}^{\left\{1^{2}\right\}} \approx T_{1 \mathrm{u}} .
\end{aligned}
$$

It is worthwhile to olsserve that for repeated irreps entering $R^{O_{h}: C_{1 h}}$ (i.e. for $\Lambda=$ $E_{\mathrm{g}}, T_{1 \mathrm{u}}$ and $\left.T_{2 \mathrm{u}}\right)$ the definite statistics $y_{0}$ is achieved by an appropriate choice of the repetition label $a \in \tilde{m}(\omega, \Lambda)$. Note also that the transitive representations $\left(R^{O_{h}: C_{4 v}}\right)_{\omega}$ for $\omega=0$ and 2 are isomorphic with respect to the geometric symmetry group $O_{h}$, but are inequivalent with respect to the Pauli group $\Sigma_{2}$ (cf. Eq. (30) with (33), (34)), The source of this difference is the fact that the orbits $\omega=0$ and $\omega=2$ consist of pairs of arms $(k, k)$ and $(k,-k)$, respectively, which yields the same geometric symmetry and distinction in statistics. 


\section{Racah recoupling matrices}

Each of coupling schemes introduced in the previous section, namely $L S$-like scheme (13), $j j$-like (16), and transitive (22), provides a complete orthonormal basis for the space of two-electron states of the considered model. Racah recoupling matrices provide an appropriate unitary transformation between any pair of these bases. We consider here only transformations between orbital parts of the two-electron wave functions in the $L S$-like and transitive schemes, and choose each basis as adopted to the Pauli group $\Sigma_{2}$ (i.e. each basis vector has a definite orbital statistics $y_{0}$ ). The Racal recoupling matrix is defined by the formula

$$
\left\langle y_{0} \omega \Lambda a \lambda \mid y_{0}^{\prime} \Lambda_{1} \Lambda_{2} \Lambda^{\prime} \lambda^{\prime}\right\rangle=\delta_{y_{0} y_{0}^{\prime}} \delta_{\Lambda \Lambda^{\prime}} \delta_{\lambda \lambda^{\prime}}\left[\omega a\left\|\Lambda y_{0}\right\| \Lambda_{1} \Lambda_{2}\right],
$$

which results from an elementary representation theory of groups (the Schur lemma for the geometric symmetry group $O_{h}$ and the Pauli group $\Sigma_{2}$ ). This formula states that elements of the transformation matrix are diagonal with respect to irreps $y_{0}$ and $\Lambda$ of the Pauli $\Sigma_{2}$ and geometric symmetry $O_{h}$ group, respectively, and is independent of a choice of basis states $\lambda$ for the irrep $\Lambda$. The symbols $\left[\omega a\left\|\Lambda y_{0}\right\| \Lambda_{1} \Lambda_{2}\right]$ form a unitary matrix labelled by $\Lambda y_{0}$, with rows and columns

\section{TABIE}

Racalı matrices $\left[\omega_{a}\left\|\Lambda_{0}\right\| \Lambda_{1} \Lambda_{2}\right]$ for the sy'stem of two electrons on a single star $p 00$. One-dimensional matrices are omitted here - they are given by Eq. (36).

a) spin singlets $\left(y_{0}=\{2\}\right)$ :

\begin{tabular}{c|c|c|c|c}
\hline \hline$A_{1 \mathrm{~g}}$ & $\{2\}$ & $A_{1 \mathrm{~g}} A_{1 \mathrm{~g}}$ & $E_{\mathrm{g}} E_{\mathrm{g}}$ & $T_{1 \mathrm{u}} T_{1 \mathrm{u}}$ \\
\hline 0 & - & $2 / \sqrt{6}$ & $-1 / \sqrt{3}$ & 0 \\
1 & - & $1 / \sqrt{6}$ & $1 / \sqrt{3}$ & $-1 / \sqrt{2}$ \\
2 & - & $1 / \sqrt{6}$ & $1 / \sqrt{3}$ & $1 / \sqrt{2}$ \\
\hline \hline$E_{\mathrm{g}}$ & $\{2\}$ & $A_{1 \mathrm{~g}} E_{1 \mathrm{~g}}$ & $E_{\mathrm{g}} E_{\mathrm{g}}$ & $T_{1 \mathrm{u}} T_{1 \mathrm{u}}$ \\
\hline 0 & - & $-1 / \sqrt{3}$ & $-2 / \sqrt{6}$ & 0 \\
1 & 1 & $1 / \sqrt{3}$ & $-1 / \sqrt{6}$ & $1 / \sqrt{2}$ \\
2 & - & $1 / \sqrt{3}$ & $-1 / \sqrt{6}$ & $-1 / \sqrt{2}$ \\
\hline$T_{1 \mathrm{u}}$ & $\{2\}$ & $A_{1 \mathrm{~g}} T_{1 \mathrm{u}}$ & $E_{\mathrm{g}} T_{1 \mathrm{u}}$ & \\
\hline 0 & - & $2 / \sqrt{6}$ & $-1 / \sqrt{3}$ & \\
1 & 1 & $1 / \sqrt{3}$ & $2 / \sqrt{6}$ \\
$\mathrm{~b}) \mathrm{spin}$ triplet $\left(y_{0}=\left\{1^{2}\right\}\right):$ \\
\hline \hline$T_{1 \mathrm{u}}$ & $\left\{1^{2}\right\}$ & $A_{1 \mathrm{~g}} T_{\mathrm{lu}}$ & $E_{\mathrm{g}} T_{1 \mathrm{u}}$ \\
\hline 1 & 2 & $-2 / \sqrt{6}$ & $1 / \sqrt{3}$ \\
2 & - & $-1 / \sqrt{3}$ & $-2 / \sqrt{6}$
\end{tabular}


labelled by $\omega a$ (the transitive scheme), and $A_{1} \Lambda_{2}$ (the $L S$-like scheme), respectively.

We present here the complete set of Racah matrices for the system of two electrons on a single star $p 00$. The one-dimensional Racah matrices are given by the formula

$$
\begin{aligned}
& -\left[1\left\|T_{2 \mathrm{~g}},\{2\}\right\| T_{1 \mathrm{u}} T_{1 \mathrm{u}}\right]=\left[1\left\|T_{2 \mathrm{u}},\{2\}\right\| E_{\mathrm{g}} T_{1 \mathrm{u}}\right]=\left[1\left\|A_{2 \mathrm{~g}},\left\{1^{2}\right\}\right\| E_{\mathrm{g}} E_{\mathrm{g}}\right] \\
& =-\left[1,2\left\|E_{\mathrm{g}},\left\{1^{2}\right\}\right\| A_{1 \mathrm{~g}} E_{\mathrm{g}}\right]=-\left[1\left\|T_{1 \mathrm{~g}},\left\{1^{2}\right\}\right\| T_{1 \mathrm{u}} T_{1 \mathrm{u}}\right] \\
& =\left[1,2\left\|T_{2 \mathrm{u}},\left\{1^{2}\right\}\right\| E_{\mathrm{g}} T_{1 \mathrm{u}}\right]=1,
\end{aligned}
$$

where all redundant $a$ labels are omitted. The multidimensional Racah matrices are collected in Table.

\section{Discussion}

We have presented here various coupling schemes and recoupling transformations for the system of two electrons confined to a single star $(p 00)$, in a way similar to Racah-Wigner calculus for multielectron atoms. In particular, we calculated a complete set of Racah recoupling matrices between the $L S$-like and transitive schemes of two-electron basis states. We pointed out natural interpretations of both schemes in terms of multipole expansions and corresponding octahedral counterparts of partial waves. It follows that our model with the star $(p 00)$ involves the monopole $\Lambda=A_{1 \mathrm{~g}}$, the quadrupole $\Lambda=E_{\mathrm{g}}$ and the dipole $\Lambda=T_{1 \mathrm{u}}$ at the single-electron level, and extends to another quadrupole $\Lambda=T_{2 \mathrm{~g}}$ and octupoles $\Lambda=A_{2 \mathrm{~g}}$ and $\Lambda=T_{2 \mathrm{u}}$ for the case of two electrons. Clearly, Wigner spherical multipoles become undistinguishable after subduction to the octahedral symmetry $O_{h}$, e.g. the same label $\Lambda=T_{1 \mathrm{u}}$ corresponds to cubic dipoles (from $l=1$ ) and octupoles (from $l=3$ ).

The tables of Racah matrices provide a transparent way of comparison of basis states within the $L S$ and transitive coupling schemes. Each table corresponds to adequate octahedral harmonic (partial wave) $\Lambda$ for the two-electron system, with an appropriate orbital statistics $y_{0}=\{2\}$ or $\left\{1^{2}\right\}$ (for spin singlets $S=0$ and triplets $S=1$, respectively). For example, generic quadrupole $\Lambda=E_{\mathrm{g}}$ for spin singlets is a superposition of 3 basis states $\Lambda_{1} \Lambda_{2}=A_{1 \mathrm{~g}} E_{\mathrm{g}}, E_{\mathrm{g}} E_{\mathrm{g}}, T_{1 \mathrm{u}} T_{1 \mathrm{u}}$ for the $L S$ case, and $\omega=1,2,3$ for the transitive scheme, the same quadrupole for spin triplets is uniqucly determined by a single basis state $\left(\Lambda_{1} \Lambda_{2}=A_{1 \mathrm{~g}} E_{\mathrm{g}}\right.$, or $\omega=1$, both cases restricted to the antisymmetric basis $y_{0}=\left\{1^{2}\right\}$ ), the octupole $\Lambda=T_{2 \mathrm{u}}$ is uniquely defined for both the singlet and triplet cases, the quadrupole $\Lambda=T_{2 \mathrm{~g}}$ exists (and is unique) for singlets only, whereas the octupole $\Lambda=A_{2 \mathrm{~g}}$ arises only for triplets, etc. Racal matrices allow us also to draw some conclusions on motion of the system in a state with definite resultant octahedral harmonic $\Lambda$. E.g. $\Lambda=A_{1 g}$, i.e. the octaliedral counterpart of completely symmetric spherical $s$-wave is in general a superposition of three $L S$ basis states $\Lambda_{1} \Lambda_{2}=A_{1 \mathrm{~g}} A_{1 \mathrm{~g}}, E_{\mathrm{g}} E_{\mathrm{g}}$, and $T_{1 \mathrm{u}} T_{1 \mathrm{u}}$. In turn, the states $\Lambda_{1} A_{2}$ are superpositions of orbits $\omega=0,1$, and 2 of the transitive scheme, thus the $L S$ state $A_{1 \mathrm{~g}} A_{1 \mathrm{~g}}$ is dominated by the orbit $\omega=0$ (with the probability $2 / 3$ ), the state $E_{\mathrm{g}} E_{\mathrm{g}}$ is characterised by equal probabilities $1 / 3$ from each orbit, whereas the state $T_{14} T_{14}$ is composed only from orbits $\omega=1$ and 
$\omega=2$, with the exclusion of the diagonal orbit $\omega=0$. Similarly, octahedral harmonics $\Lambda=T_{2 \mathrm{~g}}, T_{2 \mathrm{u}}$ for $y_{0}=\{2\}$ and $A_{2 \mathrm{~g}}, E_{\mathrm{g}}, T_{1 \mathrm{~g}}, T_{2 \mathrm{u}}$ for $y_{0}=\left\{1^{2}\right\}$ emerge from the single orbit $\omega=1$ and thus correspond to a motion in which momenta of each electron are mutually perpendicular, whereas all other harmonics admit a superposition of states with single-electron momenta being parallel or antiparallel.

\section{References}

[1] D. Loss, D.P. Vincenzo, G. Grinstein, Physica B 189, 189 (1993).

[2] M.A. Kastner, Physics Today 1, 24 (1993).

[3] The Quantum IIall Effect, Eds. R.A. Prange, S.M. Girving, Springer, Berlin 1987.

[4] IIigh Temperature Superconductivity, Ed. J.W. Lynn, Spinger, Berlin 1990.

[5] B.G. Wybourne, Spectroscopic Properties of the Rare Earths, Wiley, New York 1965.

[6] T. Lulek, in: Symmetry andStructural Properties of Condensed Matter, Eds. W. Florek, T. Lulek, M. Mucha, World Sci., Singapore 1991, p. 278.

[7] A.P. Jucys, A.A. Bandzaitis, Theory of Angular Momentum in Quantum Mechanics, Mintis, Vilnius 1965 (in Russian).

[8] B. Lulek, T. Lulek, R. Chatteryjee, J. Biel, Can. J. Phys. 63, 1061 (1985).

[9] T. Lulek, Acta Phys. Pol. A 75, 187 (1989). 